\section{Psychophysical scales of the olfactory pleasantness of homologous alcohols}

\author{
KARL E. HENION \\ University of Texas, Austin, Texas 78712
}

A category, a ratio estimation, and three magnitude scales were constructed from the observations of 10 Os who judged the pleasantness of a homologous series of n-aliphatic alcohols. Magnitude scaling indicated that pleasantness generally varies inversely with chain length, which is consistent with the findings of earlier investigators who used different methods; ratio estimation also indicated an inverse relationship. Stable magnitude scales resembled power functions with exponents of -0.262 to -0.587 ; the adjusted ratio estimation's was -0.138 . Pleasantness seemed to be unexpectedly prothetic, some evidence of which had also been reported in an earlier study involving a more diverse set of odorants. Yet in the present study the hedonic range was small and in the earlier, large. More than the size of range appears to account for the puzzling prothetic evidence.

The gradual change in the physical properties of a homologous series of alcohols as chain length increases makes them appropriate stimuli for olfactory research. Although the relation between the intensity of an alcohol and its position in a series has not been fully established (Moulton \& Eayrs, 1960), progress in this area is being made (Engen, 1965).

Less is known of the relation between the attribute of pleasantness of an alcohol and its serial position. This relation is of interest since the hedonic property of an odor stimulus may substantially influence variation in olfactory response, as Engen and McBumey (1964) have pointed out. An introspective analysis of pleasantness was done by Engen (1964) as an adjunct to a larger study involving the factor analysis of similarity judgments of a homologous series of alcohols. The results indicated that the attribute may vary inversely with chain length, pentanol excepted. The present study investigated this indication further, using direct methods of psychophysical scaling that included magnitude and ratio estimation scales. A category scale was also constructed, and it was compared with magnitude estimation scales in order to determine if the continuum of pleasantness had metathetic, which was expected, or prothetic properties.

\section{METHOD}

\section{Observers}

Ten male undergraduates volunteered to serve without pay as Os; none had participated in olfactory experiments before.

\section{Apparatus and Odorants}

The $O$ sniffed cotton saturated with a given undiluted alcohol. The cotton used was that wrapped on one end of a Q-tip.
The other cotton end was removed, and this bare end was inserted into the bottom of a small aluminum-foil-wrapped cork, big enough to be taken up easily with the fingers. After each sniff, the $O$ returned the cork with the attached Q-tip to a $10 \times 75 \mathrm{~mm}$ Pyrex test tube containing $1.5 \mathrm{cc}$ of the alcohol. When the test tube was stopped by the cork, the bottom of the cotton tip was slightly immersed in the alcohol.

The odorants were nine undiluted n-aliphatic alcohols consisting of the homologous series, ethanol $\left(\mathrm{C}_{2}\right)$ to decanol $\left(C_{10}\right)$. Seven of the nine were of extremely high purity (chromatographic or Baker-analyzed reagent grades), and the other two only slightly less pure.

\section{Procedure}

The experiments were conducted in an air-conditioned and ventilated room where the temperature was kept at $25^{\circ} \mathrm{C}$. There were five experimental conditions: three required the $O s$ to make magnitude estimations with different standards, one to make ratio estimations, and one to make category ratings. Every $\mathrm{O}$ participated in all conditions, each of which he was exposed to in a single $1-\mathrm{h}$ session, at a rate of one condition per day over 5 days, in most cases consecutive days, and in a counterbalanced order.

The $\mathrm{E}$ instructed the $\mathrm{O}$ to judge the pleasantness of the stimulus and to ignore all other attributes, such as intensity or oiliness, to judge each comparison stimulus independently of another, to sniff consistently, and to place the cotton at the same distance from his nose each time.

The standard for a magnitude estimation condition was butanol, hexanol, or octanol. The $O$ was instructed to let 10 represent the pleasantness of the standard, which he was asked to become familiar with. Including the standard as a comparison stimulus, the $E$ presented the nine comparison stimuli singly and in random sequence, employing five trials for each stimulus. The O's task was to judge the pleasantness of a stimulus by assigning a number to it proportionate to the 10 of the standard on a ratio scale. He could refer to the standard as often as he wanted and could repeat an observation provided that there was a pause of at least $45 \mathrm{sec}$ between sniffs, which was the minimal time between any two sniffs in the experiment. Duplicate sets of comparison stimuli were used in all conditions to reduce the possibility of recognition of previously presented stimuli from accidental markings on the corks or the test tubes.

In the ratio estimation condition, the $\mathrm{E}$ presented each of the 36 pairs possible from the nine comparison stimuli once, singly, and in irregular order. Half of the Os (every other one) began at one end of the order, and the other half at the other end. The O's task was to sniff the left element of a pair first, the right one nexi, and then to report which was the more pleasant and what percentage the less pleasant odorant was of the more pleasant one. Each alcohol appeared equally often on the left as on the right.

In the category estimation condition, there were two standards: The $O$ was instructed to denote the pleasantness of decanol as 2, representing a lower level of pleasantness, and of ethanol as 9 , a higher level. The $O$ 's task was to assign to each comparison stimulus an integer from 2 to 9 , inclusive, reflecting the pleasantness of the stimulus in relation to that of the two standards on an equal-interval scale. If the $O$ judged the pleasantness of a comparison stimulus to be outside this range, he could assign a number, 1 or 10 , to it. This was permitted in order to deal with possible end effects. As in each of the magnitude estimation conditions, there were 45 presentations of the nine comparison stimuli, singly presented, in random sequence, and five trials each.

\section{RESULTS}

Geometric means were computed from the 50 numbers assigned by the $O$ s to each alcohol in each magnitude condition, and were combined to generate a general function (Stevens, 1956), with butanol as standard rather than heptanol (discussed below). Engen and Lindström (1963) have described the computational procedure. The results are presented in the first column of Table 1.

The $C_{2}$ alcohol was judged the most pleasant and $C_{10}$ the least, and this result was the same for the combined magnitude 
Table 1

Scale Values of the Pleasantness of a Homologous Series of n-Aliphatic Alcohols by Three Methods

\begin{tabular}{lccc}
\hline & \multicolumn{3}{c}{ Method } \\
\cline { 2 - 4 } $\begin{array}{c}\text { Chain } \\
\text { Length }\end{array}$ & $\begin{array}{c}\text { Magnitude } \\
\text { Esti- } \\
\text { mation* }\end{array}$ & $\begin{array}{c}\text { Ratio } \\
\text { Esti- } \\
\text { mation+ }\end{array}$ & $\begin{array}{c}\text { Category } \\
\text { Rating }\end{array}$ \\
\hline C2 & 15.91 & 1.24 & 7.24 \\
C3 & 12.34 & 0.99 & 5.76 \\
C4 & 10.03 & 0.92 & 6.82 \\
C5 & 11.34 & 1.12 & 6.26 \\
C6 & 10.40 & 0.97 & 5.52 \\
C7 & 10.79 & 0.96 & 5.18 \\
C8 & 9.65 & 0.90 & 4.86 \\
C9 & 9.35 & 0.99 & 4.30 \\
C10 & 8.60 & 0.89 & 3.96 \\
\hline
\end{tabular}

*Adjusted and combined, +unadjusted, tarithmetic means.

scale and for each of the separate magnitude scales. The magnitude scales resemble power functions with negative exponents on $\left[C_{2}, C_{10}\right]$. On this interval each set of data plotted in $\log -\log$ coordinates fits a straight line; least-squares regressions produced correlation coefficients of 0.91 for the combined scale, and $0.41,0.93$, and 0.75 for the separate scales whose standards were butanol, hexanol, and octanol, respectively. The exponents of the corresponding power functions are -0.307 , and -0.587 , -0.340 , and -0.262 .

Geometric means of the 10 ratio estimates made by the Os for each of the 36 stimulus pairs were computed for the ratio estimation condition, and these means were analyzed by the matrix procedure of Ekman (1958). The resulting ratio estimation scale values are shown in the second column of Table 1. These results are consistent with those obtained by means of magnitude scaling. Again $C_{2}$ was judged most pleasant and $C_{10}$ least. As in magnitude scaling, a relative minimum occurs at $\mathrm{C}_{4}$ and a relative maximum at $\mathrm{C}_{5}$. Both scales indicate that, generally, pleasantness varies inversely with chain length on $\left[\mathrm{C}_{2}, \mathrm{C}_{10}\right]$. Although the scales form functions that are piecewise monotonic in four places, the magnitude scale shows pleasantness to be strictly decreasing on the deleted interval $\left[\mathrm{C}_{5}, \mathrm{C}_{10}\right] \sim \mathrm{C}_{7}$; the ratio estimation scale, on $\left[\mathrm{C}_{5}, \mathrm{C}_{10}\right] \sim \mathrm{C}_{9}$.

Ratio estimation scale vaiues were proportionally adjusted to the geometric means of the separate magnitude scale which had butanol as its standard, i.e., the middle standard. This was done for the purpose of comparing the adjusted ratio estimation scale with the combined magnitude estimation scale, itself constructed by means of adjustments to the butanol data. The two scales are presented in Fig. 1 as functions of chain length in $\log$ coordinates. Least-squares regression lines were fitted to both sets of data on $\left[\mathrm{C}_{2}, \mathrm{C}_{10}\right]$. The correlation coefficient of 0.91 obtained for the magnitude scale compares with 0.70 for the adjusted ratio estimation scale; the obtained slope of -0.307 for the first is less than that of -0.138 for the second.

Presented in the last column of Table 1 are arithmetic means of the 50 category estimation ratings made by the $10 \mathrm{Os}$ for each odorant.

\section{DISCUSSION}

There appears to be a division in the magnitude responses that separates the lightest alcohols from the rest in the series, since it takes the $\mathrm{C}_{3}$ or $\mathrm{C}_{2}$ alcohol to evoke a substantial difference in pleasantness. What was unanticipated was that the point of separation would occur so far to the left in the series. It was thought, based on the results of previous introspective studies (Engen, 1964; Pilgrim \& Schutz, 1957), that the Os would find $\mathrm{C}_{4}$ and $\mathrm{C}_{5}$ much more pleasant than the heavier alcohols. Otherwise, the magnitude results largely confirm those of the other studies, namely, that pleasantness generally varies inversely with chain length.

The finding that $C_{4}$ clearly does not conform (too low) to the inverse relation and that $\mathrm{C}_{7}$ does not quite (about 0.4 scale units too high) invites conjecture when considered along with the findings of other studies in which these two alcohols were part of the odorant set. The Os in a study by Kruger, Feldzamen, and Miles (1955) involving the intensity of undiluted alcohols $\left[\mathrm{C}_{3}, \mathrm{C}_{12}\right]$ found $\mathrm{C}_{4}$ to be the most intense, and this was the same conclusion of Backman (1917) based on his rule of solubility of an odorant in water and lipoids. In these cases, as in the present, the intensitive force of the molecules of the odorant may have activated trigeminal nerve endings to some small degree instead of olfactory receptors, with a consequent minor irritation and slight reduction in the perceived pleasantness of the alcohol. As for $\mathrm{C}_{7}$, Engen reported that it was the only alcohol that did not conform (too low for its serial position) to an otherwise inverse relation that was found between intensity and chain length of an undiluted $\left[\mathrm{C}_{2}, \mathrm{C}_{1} 0\right] \sim \mathrm{C}_{9}$ set; in his study, disruption in the normal breathing pattern of human infants was used as an index of olfactory intensity (Engen, 1965).

Under the assumption of a strictly decreasing function for pleasantness over the entire interval $\left[C_{2}, C_{10}\right]$ studied, it was thought that hexanol would be a suitable middle standard for generalizing the functions derived from the separate

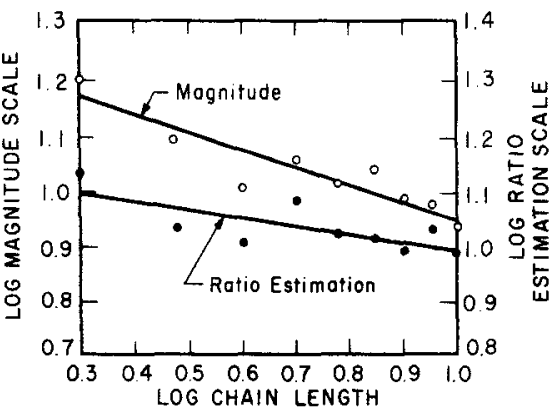

Fig. 1. Log combined magnitude estimation scale and $\log$ adjusted ratio estimation scale as functions of $\log$ chain length.

magnitude scales into a combined function. But butanol was found to be less pleasant than its ascendent neighbor in the series, hexanol, and hence the former was used as the middle standard.

Also using direct methods, Engen and McBurney (1964) had expected and did find the range of pleasantness for a wide variety of different odorants to be considerably greater than the range of intensity for a single odorant at different concentrations. The expectation in the present investigation was that the hedonic range for a homologous series of alcohols, a much more restricted variety of odorants, would probably fall in between, but in any event would likely be closer to the intensity range for a single diluted odorant than to the hedonic range of a diverse set, since over half of the odorants used exhibit a musty or oily quality. The last was confirmed: As measured on the combined magnitude scale, the largest sensation was just less than twice the smallest sensation. This compares, for example, with the subjective range of just over twice as much that was obtained with a series of geometrically diluted concentrations of $\mathrm{n}$-heptane (Engen, 1964). It also is consistent with the short subjective range of another olfactory attribute, namely, that of oiliness for a single diluted odorant (Henion, 1969).

The range of exponents of the stable power functions produced by the present magnitude scales is somewhat greater than that obtained in the case of the odor intensity for a single diluted odorant (Engen \& Lindström, 1963). This difference was not unexpected, given the especially subjective task of judging pleasantness. Also, not entirely unexpected is the lack of complete agreement between the slopes of the combined magnitude function and the ratio estimation function, where the negative slope of the first is greater than that of the second. This is not the first time that the slopes of these two 


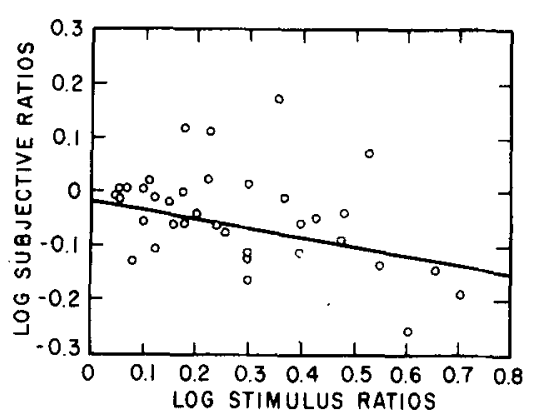

Fig. 2. Log subjective ratios as a function of $\log$ stimulus ratios.

scales have been found to vary some for a particular olfactory attribute. Engen and Lindström (1963) reported the positive slope $\left(m_{1}\right)$ of the combined magnitude scale as being somewhat greater than that $\left(m_{2}\right)$ of the ratio estimation scale in the case of the olfactory intensity of diluted amyl acetate. But in the case of the olfactory oiliness of diluted heptanol, $\mathrm{m}_{1}$ was found to be less than $\mathrm{m}_{2}$ (Henion, 1969). Little is known to account for the difference in the slopes of these kinds of scales.

Of the 36 pairs of stimuli of the ratio estimation condition, there are four pairs, for example, such that the ratio of the carbon number of each pair is in a ratio of $2 / 1$, viz, $10 / 5,8 / 4$, etc. Altogether, the 36 pairs have carbon members consisting of 26 different ratios. Corresponding to these 26 stimulus ratios were the 36 subjective ratios made by the Os. These two sets of ratios were analyzed in a log-log regression plot, presented in Fig. 2. The analysis implies internally consistent data since the least-squares line, which fits the data to a

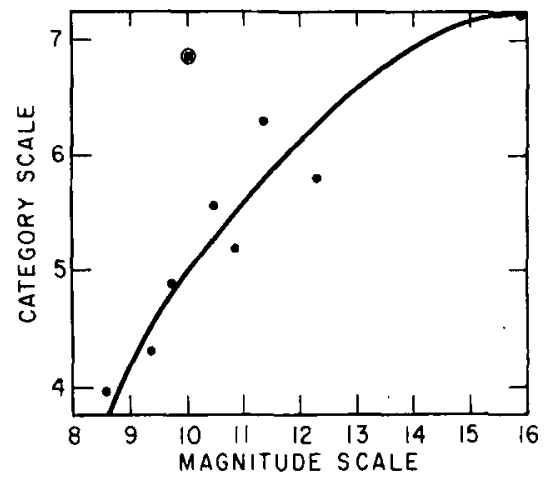

Fig. 3. Category scale as a function of combined magnitude estimation scale (fitted by eye). moderate degree $(r=0.41)$, passes near the origin with small error, generating an equation $R=1.03 S^{-0.201}$.

Criteria are available to help deal with the well-known problem of the relation between the category and magnitude scales. It was predicted that the pleasantness of the homologous series would probably constitute a metathetic continuum inasmuch as olfactory qualities were not all the same, even though the set was not particularly diverse. The absence of linearity in Fig. 3 , where the category scale is plotted as a function of the magnitude scale, suggests that the attribute may not be metathetic, although linearity is not a sufficient condition for metatheticity (Stevens \& Galanter, 1957). The circled point in the figure represents the $C_{4}$ alcohol, which constitutes the previously mentioned separating point in the magnitude judgments and does not conform to the generally negative monotonic property of the combined magnitude function. If the $\mathrm{C}_{4}$ data are removed from the analysis, then a possible interpretation is that of a function which is slightly concave downward, although $\mathrm{C}_{3}$ and $C_{7}$ are still overly transduced. This tentative conclusion is consistent with the concave-downward curve in the same kind of plot of the pleasantness of a much larger set of diversified odorants (Engen \& McBurney, 1964). Thus based on a well-known criterion, pleasantness would be characterized prothetic (Stevens \& Galanter, 1957).

To pursue the question further, the magnitude and category scales were inverted in Fig. 4, where they are plotted as functions of chain length in linear coordinates. The inversion changes each $y$-axis to a dimension of unpleasantness. Had the category scale been more concave downward than the magnitude scale, which it obviously is not, there would have existed additional grounds for the prothetic argument (Stevens \& Galanter, 1957, p. 378).

In discussing their results, Engen and McBurney (1964) suggested the notion that the size of the subjective range may be an important factor in influencing curvature. For the odorant set they had selected, the most pleasant sensation was 125 times the least pleasant sensation, yet for the present set the most pleasant was but twice the least. Thus, examples of large and small subjective ranges obtained for the same general kind of continuum

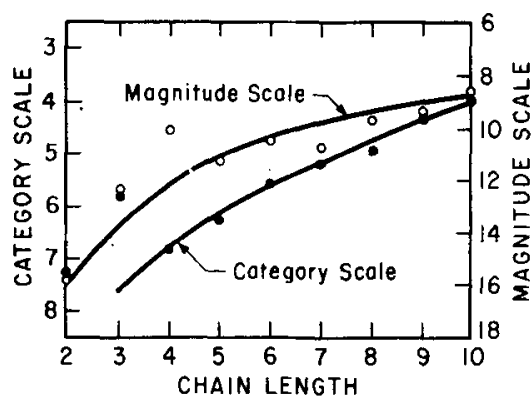

Fig. 4. Category and combined magnitude estimation scales as functions of chain length (fitted by eye).

produced somewhat similar results concerning curvature. More than subjective range appears to be involved.

\section{REFERENCES}

BACKMAN, E. L. Note sur la puissance des odeurs et leur solubilité dans l'eau et dans l'huile. Journal de Physiologie et de Pathologie Générale, 1917, 17, 1-4.

EKMAN, G. Two generalized ratio scaling methods. Journal of Psychology, 1958, 45, 287-295.

ENGEN, T. Psychophysical scaling of odor intensity and quality. Annals of the New York Academy of Sciences, 1964, 504-516.

ENGEN, T. Psychophysical analysis of the odor intensity of homologous alcohols. Journal of Experimental Psychology, 1965, 70, 611-616.

ENGEN, T., \& LINDSTRÖM, C. O. Psychophysical scales of the odor intensity of amyl acetate. Scandinavian Journal of Psychology, 1963, 4, 23-28.

ENGEN, T., \& McBURNEY, D. H. Magnitude and category scales of the pleasantness of odors. Journal of Experimental Psychology, $1964,68,435.440$

HENION, K. E. Subjective range of the odor oiliness of heptanol. Journal of Experimental Psychology, 1969, 82, 515-519.

KRUGER, L,, FELDZAMEN, A. N., \& MILES, W. R. Comparative olfactory intensities of the aliphatic alcohols in man. American Journal of Psychology, 1955, 68, 386-395.

MOULTON, D. G., \& EAYRS, J. T. Studies in olfactory acuity. II. Relative detectability of n-aliphatic alcohols by the rat. Quarterly Journal of Experimental Psychology, 1960, 12, 99-109.

PILGRIM, F. J., \& SCHUTZ, H. G. Measurement of the qualitative and quantitative attributes of flavor. In Quartermaster Food Container Institute, Chemistry of Natural Food Flavors, 1957, 47-55.

STEVENS, S. S. The direct estimation of sensory magnitudes-loudness. American Journal of Psychology, 1956, 69, 1.25.

STEVENS, S. S., \& GALANTER, E. H, Ratio scales and category scales for a dozen perceptual continua. Journal of Experimental Psychology, 1957, 54, 377-411.

(Accepted for publication July 12, 1970.) 